\title{
Unité proposée basée sur l'approche interculturelle pour le développement de la compréhension du concept de la gastronomie française chez les étudiants des facultés de Pédagogie
}

\author{
Sally Mohamed Hélmi Ali Elddine
}

\section{Introduction}

L'apprentissage d'une langue étrangère permet aux étudiants d'acquérir de compétences linguistiques et communicatives. Il permet également à l'usager d'une langue étrangère de développer son sens critique, de construire de nouvelles relations, de valoriser sa propre culture et de construire une relation de respect pour la culture de l'autre (Vanessa, 2012: 1).

Le concept d'interculturel s'inscrit dans un champ plus vaste; il inclut bien sûr des relations, des interactions entre des cultures différentes générées par des rencontres ou des confrontations, qualifiées d'interculturelles (Tourev, 2006: 24).

L'interculturalité peut contribuer à surmonter le sectarisme face à la culture de l'autre, mais peut aussi aider à déconstruire la crainte d'établir des relations avec des personnes qui ont des comportements différents ou des valeurs, des attitudes et des pensées différentes.

Cette relation démontre également une véritable intégration interculturelle, c'est-à-dire l'instauration d'une véritable interaction et coopération entre les individus de différents mondes culturels. Les relations interculturelles impliquent un effort pour partager les expériences anciennes et les récentes, afin de construire de nouvelles significations, (Vanessa,2012: 7).

Le travail sur la culture en classe de langue amène à prendre en compte non seulement des cultures sociales mais des cultures d'enseignement et des cultures d'apprentissage, avec l'application de l'approche interculturelle.

La finalité principale de la pédagogie interculturelle ayant pour but d'apprendre à des personnes de cultures différentes à communiquer entre elles et à mieux se connaître. Il est important d'apprendre à mettre à profit la diversité culturelle croissante des sociétés, la dynamique inhérente à chaque culture, afin de créer des synergies pour inventer de nouvelles façons de vivre ensemble et d'organiser les sociétés modernes (Yéo,2012: 2)

La Pédagogie interculturelle se fonde absolument sur le respect des porteurs de culture. GohardRadenkovic, (2005: 23), spécialiste dans l'étude de l'évolution de la

1-culture en pédagogie, considère que la culture se déroule en trois phases :

Premièrement, on l'a appelée l'étape extralinguistique ; il s'agit 
d'un enseignement de la culture tout en l'articulant autour de l'opposition : langue et civilisation. Deuxièment, on l'a appelée l'étape intralinguistique ; à ce stade, on s'est aperçu qu'enseigner une langue, c'est enseigner une civilisation. En revanche, avec la naissance des sciences sociales, on a assisté à une conception beaucoup plus large de la place de la culture s'agissant d'une nouvelle dimension dite interculturelle, cette dernière a marqué la troisième étape que l'on a appelée l'étape ethnocommunicative.

Le but essentiel de l'enseignement de la culture, c'est de fournir aux étudiants (futurs enseignants) une culture indispensable à la connaissance des pays étudiés et à la compréhension des oeuvres littéraires qui sont proposées par ailleurs.

La gastronomie est un élément essentiel de la culture des français. La gastronomie représente l'art d'utiliser la nourriture pour créer le bonheur. Cela signifie l'art du bien manger et du bien boire.

Churchill a montré que la gastronomie française jouit d'une tradition pluriséculaire de raffinement et d'excellence. Les Français n'en font pas moins preuve d'une curiosité gourmande pour les cuisines du monde entier et demeurent attachés au plaisir d'une table conviviale, où l'on aime autant manger que converser, éventuellement même de ce qu'on mange.

2-Position du problème de la recherche :- recherche, la chercheuse en décrit la position du problème de la recherche.

Premièrement, l'espace limité alloué à ce cours dans le programme de formation des futurs enseignants de FLE à la faculté de Pédagogie, Université de Mansourah.

Deuxièmement, la chercheuse a entrepris, comme il est d'usage, une lecture critique du programme de formation des futurs enseignants de FLE, à la faculté de Pédagogie, Université de Mansourah surtout les cursus de la culture. Le programme de formation est peu marqué par la diversité interculturelle.

Troisièmement, le niveau culturel des futurs enseignants est faible ; ceci est prouvé par les résultats du test effectué par la chercheuse pendant le mois de février 2017.

Le test appliqué aux étudiants de la 2ème année (30 étudiants) est composé de six questions principales et chaque question se subdivise en questions. Les résultats les ont beaucoup démarqués:

$80 \%$ des étudiants de la deuxième année de la faculté de Pédagogie, Université de Mansourah n'ont pas de bonnes connaissances sur la gastronomie française.

$90 \%$ des étudiants de la deuxième année de la faculté de Pédagogie, Université de Mansourah n'ont pas de bonnes connaissances sur les repas essentiels en France.

$70 \%$ des étudiants de la deuxième année de la faculté de Pédagogie, Université de Mansourah 
n'ont pas de bonnes connaissances sur la cuisine française et la recette des plats français principales.

Quatrièmement, très peu de travaux récents, à notre connaissance, ont cherché au niveau des futurs enseignants de FLE en culture française contemporaine.

Après avoir donné ce bref exposé de quelques aspects théoriques de l'interculturel, la question de sa mise en application est incontournable: comment traduire ce concept en unité pour le cours de la culture française? Cependant, il est difficile d'imaginer qu'un étudiant égyptien devienne un enseignant interculturel sans avoir acquis des connaissances sur la culture française et sans être passé par la comparaison entre la culture égyptienne et la culture française.

\section{3- Problématique de la recherche}

La problématique principale de la recherche réside dans la faiblesse du niveau des étudiants de la deuxième année du département de français à la faculté de Pédagogie, Université de Mansourah en culture française.

Ce problème pourrait se résumer en question suivante:

Quelle est l'efficacité de l'unité proposée basée sur l'approche interculturelle pour le développement de la compréhension du concept de la gastronomie française chez les étudiants des facultés de Pédagogie?

Ce sont deux questions issues de la question principale:

2-Comment élaborer l'unité proposée basée sur l'approche interculturelle pour le développement de la compréhension du concept de la gastronomie française chez les étudiants du département de Français - facultés de Pédagogie?

3. Quelle est l'efficacité de l'unité proposée basée sur l'approche interculturelle pour le développement de la compréhension $\mathrm{du}$ concept de la gastronomie française chez les étudiants de départements de Français - facultés de Pédagogie?

\section{3- Hypothèse de la recherche :}

La recherche vise à vérifier l'hypothèse suivante :

Il y a des différences statistiquement significatives entre la moyenne de notes des étudiants de l'échantillon de recherche au pré- post test de la gastronomie française en faveur du post-test.

\section{4- Objectifs de la recherche :}

- Développer la compréhension du concept de la gastronomie française chez les étudiants de la deuxième année du département de Français facultés de Pédagogie.

- Vérifier l'efficacité de l'unité proposée basée sur l'approche interculturelle pour le 
développement de la compréhension $\mathrm{du}$ concept de la gastronomie française chez les étudiants de la deuxième année du département de Français - facultés de Pédagogie.

\section{5- Importance de la recherche:}

- Cette recherche contribue au développement de la compréhension $\mathrm{du}$ concept de la gastronomie française chez les étudiants des facultés de Pédagogies en Egypte.

- Présenter aux enseignants du FLE une unité basée sur l'approche interculturelle pour le développement de la compréhension de la gastronomie française.

- Entraîner les enseignants à l'utilisation de l'approche interculturelle dans l'apprentissage /enseignement du FLE.

\section{6- Délimites de la recherche:-}

Cette recherche se limite à:

- Un échantillon d'étudiants de la deuxième année du département de français - faculté de Pédagogie, Université de Mansourah.

- Une unité proposée de deux heures hebdomadaires dans le cadre du cours de formation en culture (la gastronomie française).

\section{7- Méthodologie de la recherche :-}

La chercheuse utilise la méthode descriptive -analytique pour passer en revue le cadre théorique de la recherche. Elle utilise aussi la méthode quasi-expérimentale (désigne d'un seul groupe) pour mesurer l'efficacité de l'unité proposée basée sur l'approche interculturelle pour le développement de la compréhension $\mathrm{du}$ concept de la gastronomie française chez les étudiants du département de français, faculté de Pédagogie- Université de Mansourah.

\section{9- Outils de la recherche :}

1- Questionnaire de la gastronomie française.

2- Test de la gastronomie française.

\section{0-Procédures de la recherche :}

Pour répondre aux questions de la recherche, la chercheuse va suivre les étapes suivantes:

1-Passer en revue la littérature qui concerne les variables de la recherche: l'approche interculturelle et la gastronomie française.

2-Elaborer une liste des sous-concepts du concept de la gastronomie française chez les étudiants de la deuxième année du département de Français, faculté de PédagogieUniversité de Mansourah.

3-Elaborer l'unité proposée basée sur l'approche interculturelle en vue de développer de la compréhension du concept de la gastronomie française chez les étudiants du département de Français, faculté de PédagogieUniversité de Mansourah.

4-Elaborer un test afin de mesurer le niveau de la compréhension du concept de la gastronomie française chez les étudiants de la deuxième année du département de Français, faculté de Pédagogie - université de Mansourah.

5-Présenter les outils de la recherche aux spécialistes du FLE pour vérifier leur validité apparente. 
6-Modifier les outils de la recherche selon les points de vue du jury.

7-Standardiser les outils de la recherche.

8-Choisir l'échantillon de la recherche parmi les étudiants de la deuxième année, département de français, faculté de Pédagogie-Université de Mansourah.

9-Appliquer le pré-test à l'échantillon de l'étude.

10- Enseigner l'unité proposée à l'échantillon de la recherche.

11-Appliquer le post-test à l'échantillon de la recherche en vue de vérifier l'efficacité de l'unité proposée.

12. Analyser statiquement les résultats de la recherche.

13. Interpréter les résultats de la recherche.

14. Présenter les recommandations et les suggestions de la recherche.

\section{1- Terminologies de la recherche :-}

\section{La culture}

L' UNESCO défini la culture de cette manière-ci

« La culture, dans son sens le plus large, est considérée comme l'ensemble des traits distinctifs, spirituels et matériels, intellectuels et affectifs, qui caractérisent une société ou un groupe social. Elle englobe, outre les arts et les lettres, les modes de vie, les droits fondamentaux de l'être humain, les systèmes de valeurs, les traditions et les croyances. »

La chercheuse définit la culture comme ensemble des connaissances acquises qui permettent à l'étudiant de développer le sens critique, le goût et le jugement. Elle englobe outre les traditions, institutions et modes de vie par lesquels une personne ou un groupe exprime son humanité et les significations qu'il donne à son existence et à son développement.

\section{L'interculturalité:}

Un mode particulier d'interactions et d'interrelations qui se produisent lorsque des cultures différentes entre en contact ainsi que l'ensemble des changements et des transformations qui en résultent (Clanet, 1990 :22). Il s'agit deux cultures véhiculées par la langue maternelle et la langue étrangère.

L'interculturalité selon la chercheuse se manifeste par la rencontre de l'autre, comment vivre à ses côtés, échanger et partager. Elle sera l'occasion de la reconnaissance des différentes langues, histoires, religions, traditions, modes de vie, ainsi que toutes les particularités attribuées aux cultures francoégyptienne, tout en démontrant la reconnaissance et la compréhension de leur culture et en respectant leurs différences culturelles.

Al'heure de la multiplication des méthodes, des stratégies, et des approches d'enseignement, l'approche de l'interculturel fait désormais partie des champs de la didactique du FLE.

\section{La gastronomie}

Est l'ensemble des règles fluctuantes, selon pays, classes sociales et modes qui définissent l'art de faire bonne chère c'est-à-dire faire bon accueil.( wikipedia). 
La définition que la chercheuse adopte.

Ensemble des arts au sujet de la cuisine que le citoyen français donne à son existence actuelle et à son développement.

\section{L'unité}

C'est un cadre méthodologique qui va permettre de structurer l'enseignement d'une activité physique et sportive pour concevoir des situations pédagogiques articulées entre elles, et de veiller à l'enchaînement chronologique cohérent de ces différentes séquences en fonction d'objectifs clairs dans une perspective finalisée. (Education physique et sportive)

La chercheuse définie l'unité comme un ensemble de séances comprenant des activités d'apprentissage qui sont regroupées pour poursuivre des objectifs communs et constituent un ensemble pédagogique au niveau des acquis d'apprentissage attendus.

\section{Deuxième chapitre: Cadre} théorique de la recherche

\section{Introduction:}

La langue est une manière de parler, de s'exprimer et de communiquer avec les individus entre eux. Aujourd'hui, il est important d'apprendre plusieurs langues étrangères. Celles-ci permettent de découvrir de nouvelles cultures et ouvrent les portes vers des pays différents du nôtre. Elles permettent également de mieux appréhender les richesses de notre propre identité culturelle et d'avoir conscience des différences entre chaque nation.

Fathy a constaté" Afin d'atteindre un niveau satisfaisant dans l'apprentissage d'une langue étrangère, il faut pouvoir parvenir à penser dans cette langue. Et ceci ne peut s'acquérir que par l'étude approfondie de la culture du pays de cette langue".(Fathy (2017:1).

En outre, l'enseignement de la culture est un des moyens les plus efficaces pour motiver les apprenants de la langue étrangère. Pour développer les langues étrangères, il faut réaliser les objectifs et les compétences de savoir, savoir-être, etc. Pour Porcher, il a montré "une langue est toujours un produit culturel marqué de part de culture et enseigner l'une sans l'autre serait les rendre infirmes".(Porcher, 2004).

\section{Objectifs d'apprentissage de la langue française}

Le Bureau de Soutien à l'Enseignement (2018:1) formule des objectifs d'apprentissage qui permettent de préciser les connaissances à acquérir et les compétences à développer par les étudiants au terme d'une activité d'apprentissage. Ils interviennent dès le début du processus de planification d'un cours. Par souci de cohérence, le choix des évaluations, des activités d'enseignement-apprentissage et l'organisation du contenu du cours découlent des objectifs d'apprentissage. Ces objectifs facilitent le processus de communication entre l'enseignant et les étudiants en précisant 
l'apprentissage à faire, les actions à accomplir ou les performances à atteindre. L'étudiant connaît précisément ce qui est attendu de lui.

\subsection{Objectifs socio-culturels:}

L'objectif socio-culturel se constitue en savoirs, savoir-être, savoir-faire, compétences culturelles et interculturel.

1- Savoirs: c'est-à-dire découvrir, situer et appréhender des connaissances organisées sur un sujet.

2- Savoir-faire, savoir-être sont soulignés à découvrir, apprendre et acquérir des aptitudes à faire et à être dans un contexte. Ils résument en Découvrir, Etre capable de, rituel.

3- Les compétences culturelles comprennent la mémoire c'est-àdire tout ce qui a construit en histoire et au passé d'une culture comme l'immigration et les fêtes calendaires. En addition des échanges c'est-à-dire tout ce qui a traité aux échanges économiques et leurs implications sociales. Quant au lien social, il contient la violence, le fait religieux, le multiculturalisme, etc.

Cellier aussi présente les domaines de l'objectif culturel qui englobent les éléments culturels c'està-dire la civilisation comme l'alphabet et les différentes graphies, la personne comme (corps, vêtement), la famille et la maison, les animaux familiers, l'école en France (l'environnement scolaire, le matériel, les règles en usage...), les habitudes alimentaires, les loisirs et sports, etc. (Cellier, 2008:23)

4- Quant à objectif interculturelle: il est partie de l'objectif socioculturel et signifie prendre conscience de la différence de procédures, de réactions, de modes d'action entre le contexte français et les contextes des cultures d'origine des apprenants.(Catmar, 2014: 2).

\section{2-Premier volet: L'approche interculturelle}

\subsection{Apparition et définitions de l'interculturalité}

Comme le souligne Françoise Lorcerie(2002, 22:39): Le concept« interculturel » a apparu dans le BOEN à la fin des années soixante-dix. Il constitue une nouvelle ligne pédagogique supposée favoriser l'intégration des enfants immigrés. Cependant, si la pédagogie interculturelle était l'objet de deux expérimentations pilotes sous la direction des écoles de 1976 à 1982, elle ne dotait jamais d'instructions concrètes pour sa mise en oeuvre». En effet, les termes comme " éducation interculturelle » et « interculturalité » n'apparaissent que très peu dans les textes officiels. Cependant on observe un développement dans les nombres d'étudiants issus de différentes cultures et c'est cela qui va traduire l'émergence de la notion d'interculturalité. Effectivement, à partir de 1970, plusieurs mesures ont été instituées pour favoriser la scolarisation d'étudiants étrangers.

$$
\text { Puren (2010:1) définit }
$$

l'interculturel comme les 
représentations, les savoirs, les savoirêtre, les savoir-faire et le savoirapprendre. L'interculturel détermine comme un constituant du vivre ensemble démocratique et co-culturel qui aident les personnes de cultures différentes à agir en commun.

D'autre part, l'approche interculturelle pour la didactique de langues signifie que « c'est faire acquérir à l'apprenant une compétence aussi bien interculturelle que linguistique; le préparer à des relations avec des personnes appartenant à d'autres cultures; permettre à l'apprenant de comprendre et d'accepter ces personnes 'autres' en tant qu'individus ayant des points de vue, des valeurs et des comportements différents; enfin, aider l'apprenant à saisir le caractère enrichissant de ce type d'expériences et de relations " (Byram et d'autres, 2002:11).

\subsection{Les objectifs de l'approche}

\section{interculturelle}

Le Cadre européen commun de référence pour les langues limite quatre savoir-faire interculturels comme suit:

1-a capacité d'établir une relation entre la culture d'origine et la culture étrangère.

2-la sensibilisation à la notion de culture et la capacité de reconnaître et d'utiliser des stratégies variées pour établir le contact avec des gens d'une autre culture ;

3-la capacité de jouer le rôle d'intermédiaire culturel entre sa propre culture et la culture étrangère et de gérer efficacement des situations de malentendus et de conflits culturels;

4-la capacité à aller au-delà de relations superficielles stéréotypées. (Catroux:2009:4).

\subsection{Compétences interculturelles}

\subsubsection{Définition de la compétence interculturelle}

La notion de la compétence interculturelle représente une importance croissante chez les chercheurs et praticiens spécialistes de l'expatriation (Barmeyer et Cerdin, 2008: 2).

C'est une notion aux contours souvent flous qui regroupe toutes les attitudes, traits de personnalité, connaissances et aptitudes qui permettent à l'individu de faciliter sa communication ou son interaction avec des individus venant d'autres environnements culturels (Ogay, 2000).

\subsubsection{Les \\ interculturelles}

Les compétences interculturelles sont de plus en plus essentielles pour quelques causes.

D'abord, beaucoup de futurs professionnels, leaders d'opinion et les grandes organisations multinationales, composent quotidiennement avec la diversité culturelle. Puis, nous, comme une société d'accueil et d'un pays

industrialisé, nos étudiants ont besoin de développer des compétences interculturelles qui les aideront à réfléchir sur les grands enjeux 
internationaux en tenant compte de divers points de vue.

Par conséquent, Deardorff (2011: 26) a préparé une liste finale des aptitudes et des compétences conçues comme indispensables pour acquérir des compétences interculturelles, comprenant les cinq rapports régionaux préparés pour l'UNESCO.

Tableau 1: Deardorff. (2011).Liste des compétences interculturelles.

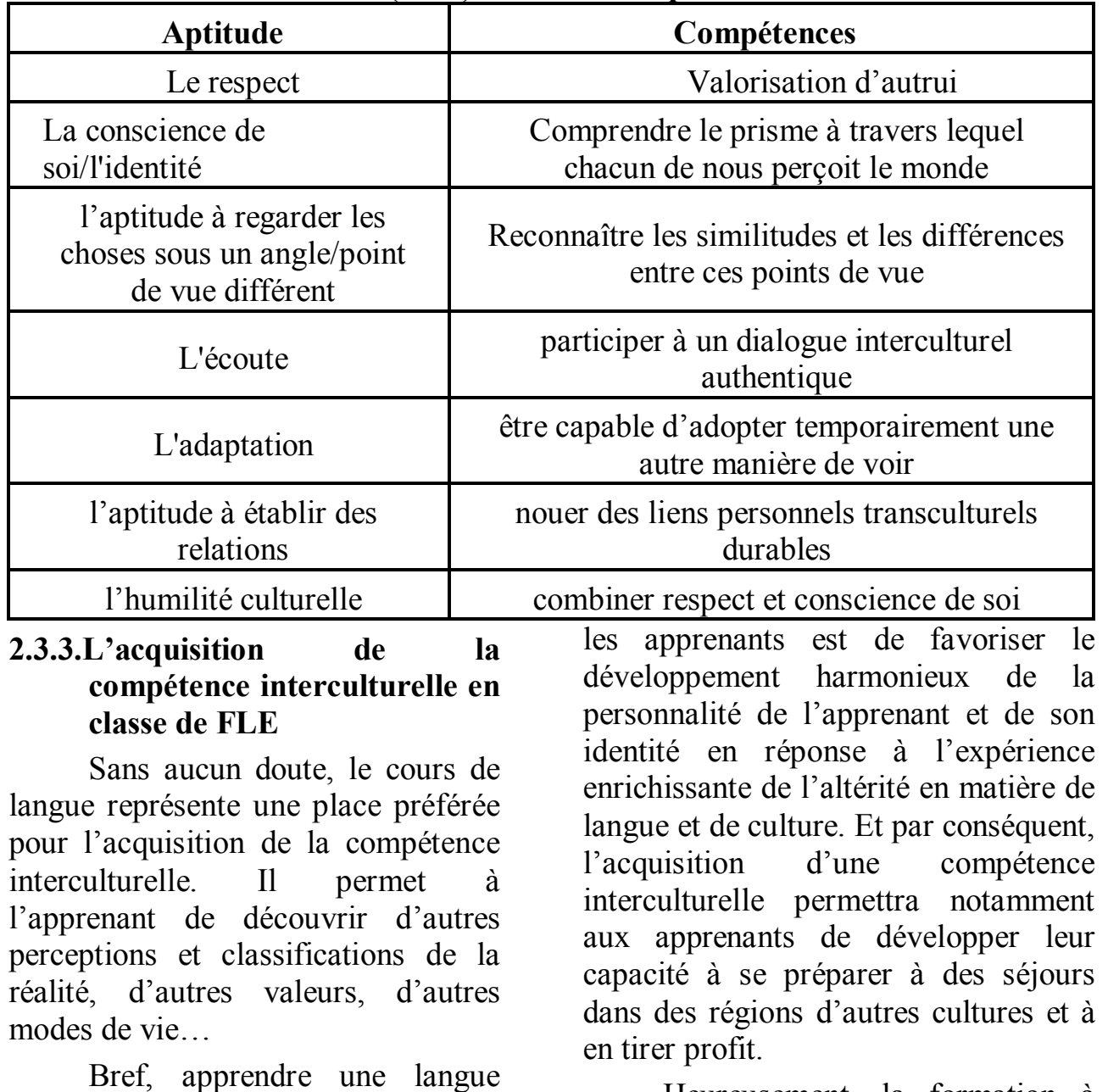
étrangère, cela signifie entrer en contact avec une nouvelle culture" (Myriam Denis, 2000: 62).

Le Cadre Européen indique qu'un des objectifs de l'acquisition d'une compétence interculturelle dans l'enseignement des langues pour

Heureusement, la formation à l'interculturel n'a pas seulement pour objectif de permettre aux apprenants de mieux maîtriser la langue étrangère dans ses dimensions linguistiques et culturelles, elle permet également de revaloriser la finalité éducative de 
l'école (remise en cause des stéréotypes sociaux, lutte contre la xénophobie et le racisme, respect de l'autre, ouverture à l'altérité) désormais élargie à un projet humaniste à l'échelle du monde (compréhension entre les peuples, enrichissement mutuel...). (Maga, 2003:4).

Donc, L'interculturel devient non seulement une des caractéristiques des programmes de formation, mais également comme une source centrale de la formation des enseignants de FLE.

\section{3-Deuxième volet: La gastronomie française}

\subsection{Définition de la gastronomie francaise}

La gastronomie française est un des concepts culturels français importants. Il signifie l'art d'utiliser la nourriture pour créer le bonheur.

La cuisine française est connue dans le monde entier. Les Français sont fiers et très attachés à leur patrimoine culinaire. Ils pensent souvent que leur cuisine est la meilleure du monde car elle est faite à partir de produits du terroir, qui sont de qualité et très diversifiés, comme la truffe, le foie gras, les fromages, le vin, etc. De nombreux produits ont le label AOC (Appellation d'Origine Contrôlée) ce qui certifie leur origine géographique ainsi que leur qualité (on ne fait pas de camembert à Marseille ou de champagne à Lyon!). C'est cela qui fait la richesse de la gastronomie et inspire depuis toujours les grands chefs étoilés. (Astirmays: 2016).
Tout le monde sait que les Français sont gourmets et maîtres de l'art de cuisine. C'est un composant intégrant de la culture française. Pour la plupart des Français, la cuisine est un des plaisirs essentiels, ils aiment se rencontrer en famille pour participer à un bon repas et pour passer plusieurs heures à table. Le vrai repas en France, ça ne signifie pas seulement manger, c'est une partie indivise de la vie culturelle. C'est le temps pour se réunir. Il faut dire que ce thème est aussi très utilisable en pratique pédagogique. (Brno: 2010).

3.2.Stratégies pour l'enseignement de la gastronomie francaise

La séquence pédagogique comprend 3 étapes pouvant correspondre à trois ou quatre séances de cours.

Étape1: Introduire la notion de cliché, de stéréotype, notamment pour les cuisines du monde.

On a besoin de la salle de classe avec vidéoprojecteur ou salle multimédia.

Activité1: échanges oraux et prise de notes.

Activité2: compréhension audiovisuelle.

Étape2:Prendre contact avec la géographie des régions françaises et des produits régionaux (22 régions de France métropolitaine et 5 régions d'outre-mer). françaises.

Activité3: revoir les régions

Activité4:consulter une carte de France des plats régionaux. 
Étape3: Prendre contact avec les recettes de cuisine.

Activité5: étudier la présentation d'une recette.

\section{Troisième}

\section{Méthodologie de la recherche}

Ce chapitre porte deux axes. Le premier axe comprend les outils et les matériels de la recherche: la grille des concepts culturels français, le test et l'unité proposée. Le deuxième axe constitue les procédures de l'étude expérimentale comme suit:

1- Elaborer une grille des sousconcepts de la gastronomie française utiles aux étudiants de la deuxième année de faculté de Pédagogie Université de Mansourah.

2- Elaborer un pré/post test visant à mesurer le concept de la gastronomie française chez les mêmes étudiants.

3- Elaborer une unité proposée basée sur l'approche interculturelle pour le développement de la compréhension du concept de la gastronomie française chez les étudiants des facultés de Pédagogie-université de Mansourah.

4- Elaborer un guide de l'enseignante.

5- Elaborer un guide de l'étudiant.

\section{6-Etapes de l'étude expérimentale:}

6.1. Choisir de l'échantillon de la recherche.

\subsection{Appliquer le pré-test de la gastronomie française.}

6.3. Enseigner l'unité proposée basée sur l'approche interculturelle pour le développement de la compréhension du concept de la gastronomie française chez les étudiants des facultés de Pédagogie- université de Mansourah.

\subsection{Appliquer le post-test de la gastronomie française.}

\section{Quatrième chapitre: Résultats de la recherche}

\section{I-Analyse statistique des résultats de la recherche}

La chercheuse a utilisé le test "T" afin de signifier les différences entre la moyenne des notes de l'échantillon de l'étude au pré/post-test de la gastronomie française. C'est clair que toutes les valeurs de "T" sont statistiquement significatives à 0,05 pour la post-application, cela indique un développement remarquable aux résultats du concept de la gastronomie française parmi les étudiants de deuxième année de la langue française en utilisant l'approche interculturelle.

Donc, il y a des différences statistiquement significatives au niveau de 0.05 entre la moyenne des notes de l'échantillon de l'étude au pré/post-test de la gastronomie française. La moyenne obtenue au pré-test est $(\mathrm{M}=$ 9.7667) et le standard de déviation est ( Std. Deviation $=1.65432$ ). Tandis que la moyenne obtenue à la postapplication $(\mathrm{M}=40.0667)$ et le standard de la déviation est (Std. Deviation $=2.37709$ ).

Ainsi, la valeur du test $\mathrm{T}$ est (60.489). Cela indique que la taille de 
l'effet est grande et que l'unité est efficace et valable. Comme il se montre aussi que les valeurs de la taille de l'effet sont grandes et plus que 0.15 . En indiquant que la taille de l'effet de l'approche interculturelle dans le développement de la compréhension du concept de la

De ce qui précède, on peut résumer que le degré de la compréhension du concept de la gastronomie française est élevé en faveur de la post application parce que les étudiants de la deuxième année au pré application n'ont pas encore connu d'informations autour de la gastronomie française. Mais après l'application de l'unité proposée, les étudiants deviennent avoir les informations à propos $\mathrm{d}$ de la gastronomie française et par conséquent, le résultat devient grand.

Les résultats de moyennes des notes de l'échantillon de recherche aux pré/post-applications et aussi le nombre global peuvent être représentés dans le diagramme suivant:

Diagramme No.(1) La moyenne des notes à la pré/post-application du test à la gastronomie française(global)

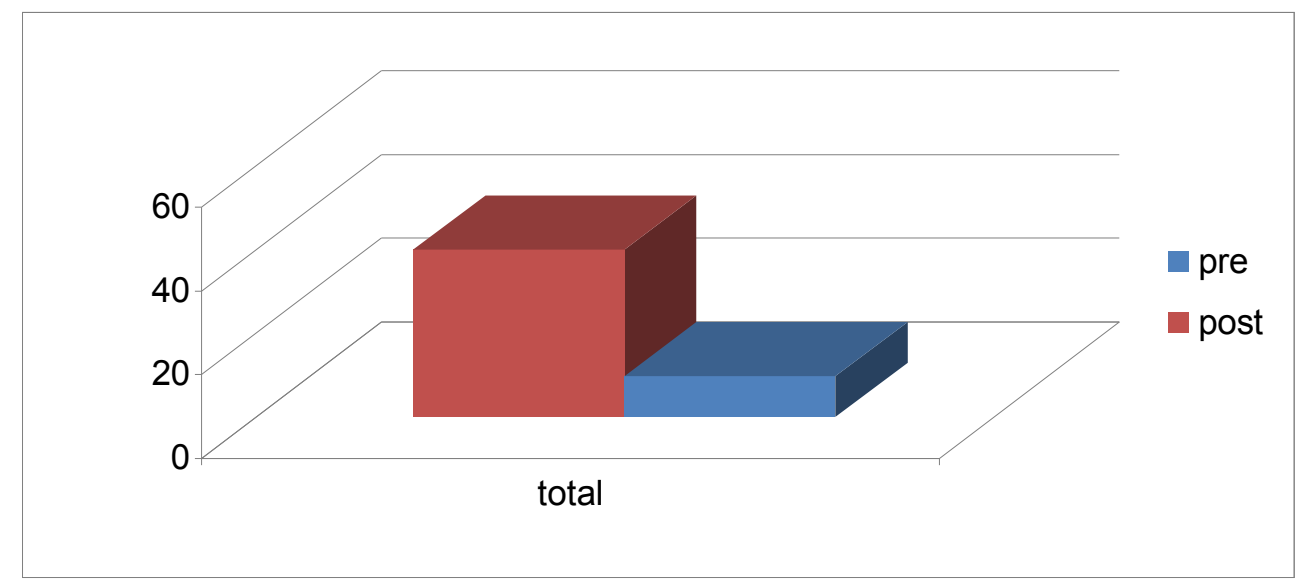

\section{Commentaire sur le diagramme:}

$\mathrm{Ce}$ diagramme résume le tableau précédent et montre la comparaison entre la moyenne des notes des étudiants au post-test et qui représente plus essentielle que celle de leurs note au pré-test en ce qui concerne la gastronomie française. Il est remarquable aussi que le résultat à la post- application est élevé et cela signifie l'efficacité de l'approche interculturelle pour développement de la compréhension de la gastronomie française chez les étudiants des facultés de PédagogieUniversité de Mansourah. Donc, l'hypothèse, qui déjà mentionnée, est valide.

hypothèses de la recherche, la chercheuse présente l'interprétation des résultats de sa recherche et à la fin, elle suggère les recommandations, 
ainsi que des pistes pour des recherches futures.

\section{II- Interprétation des résultats}

Après avoir vu les résultats obtenus de la post-application et cela après l'enseignement de l'unité proposée, il est clair qu'il y a un développement remarquable dans la performance des étudiants en ce qui concerne le concept de la gastronomie française chez les étudiants de la deuxième année universitaire à la faculté de Pédagogie.

L'approche interculturelle joue un rôle très important au développement de la compréhension $\mathrm{du}$ concept de la gastronomie française. Les recherches et les mémoires précèdantes ont confirmé son efficacité comme l'étude de Badry(2017) qui a mesuré l'efficacité d'un programme proposé basé sur l'approche interculturelle dans le développement de quelques compétences de la compréhension écrite chez les futurs enseignants de FLE. On est accord aussi avec l'étude de Elfakharany (2017) qui a développé des compétences linguistiques et communicatives des futurs-enseignants de français langue étrangère de la faculté de Pédagogie à la lueur de l'approche interculturelle via Internet.

D'ailleurs, l'étude de Darwich(2004) qui a signalé au développement culturel des futurs enseignants à la lueur de l'approche interculturelle.
Ces résultats peuvent être interprétés comme suit:

1- Les stratégies de l'enseignement à la lumière de la gastronomie française sont convenables et aident à la compréhension du contenu de la leçon.

2- Les étudiants interagissent de bonne manière en classe de FLE et ils aiment apprendre ainsi qu'ils ont la curiosité de connaître la culture française.

3- Une approche comme l'approche interculturelle joue un rôle très essentiel vu qu'elle crée chez l'étudiant une atmosphère de l'ouverture culturelle entre les peuples et les pays. Aussi, l'approche interculturelle joue un rôle préventif contre le racisme et l'exclusion.

4- Les activités de l'apprentissage et de l'évaluation sont variées et comprennent les questions, les questionnaires autour de notre culture et la culture de l'autre pays

On peut conclure que les résultats obtenus sont encourageants et que l'unité élaborée par la chercheuse a atteint sa fin.

\section{III- Recommandations de la recherche}

A la lueur des résultats de la recherche, on recommande de:

1- Entraîner les étudiants de la deuxième année, section de français d'appliquer le concept de la gastronomie française dans leur vie quotidienne.

2- Apprendre les étudiants le concept de la gastronomie 
française à partir du premier cycle jusqu'au quatrième cycle.

3- Aider les étudiants à aller à la bibliothèque pour chercher des informations autour de la culture française.

4- Créer les lieux et les activités appropriés pour conserver les informations d'une manière simple et facile dans l'esprit à titre d'exemple des chaises sous forme de circulaires et des tableaux intelligents.

5- 5-Favoriser le climat adéquat qui se réalise l'apprentissage culturel.

6- 6-Il faut permettre aux étudiants du cycle secondaire de savoir quelques notions culturelles de la société française.

\section{IV-Suggestions e la recherche}

D'après les résultats obtenus de la recherche, la chercheuse amène aux suggestions suivantes:

1-Utilisation de l'hypertextualité basée sur l'approche interculturelle pour le développement de la compréhension de la culture française chez les étudiants de FLE.

2-Développement du curriculum de la langue française à la lumière de l'approche interculturelle chez les étudiants du cycle secondaire.

3-Efficacité d'un programme proposé basé sur les intelligences multiples pour le développement des quelques concepts culturels français chez les étudiants des facultés de Pédagogie.
4-Unité proposée à l'utilisation des programmes électroniques pour le développement du contenu culturel chez les étudiants du cycle secondaire.

5- Utilisation de la méthode montessori pour développer la compréhension de la gastronomie française chez les enfants.

6-Unité proposée basée sur l'approche interculturelle pour l'acquisition des procédés de la sémiologie utilisée à la communication nonverbale chez les élèves.

7-Efficacité du jeu de rôles pour le développement de l'utilisation des réseaux sociaux chez les étudiants des facultés de Pédagogie.

\section{Bibliographie}

- AbdElhadi, R.(2014).Activités langagières pour améliorer l'acquisition de la langue française chez les élèves du cycle secondaire Azharian et leur attitudes envers la matière. Thèse de Magistère. Université de Tanta, faculté de pédagogie, département des Curricula et de Méthodologies.

- Anthropos.(2012). L'histoire interculturelle des sociétés. Exploration interculturelle et science sociale. Paris,p.312.

- Astirmays. ( 2016). Cuisine française. France:Paris. Disponible en ligne sur https://fr.wikipedia.org/wiki/Cuisi ne fran $\% \mathrm{C} 3 \% \mathrm{~A} 7 \mathrm{aise}$

- Badary, E.(2017).Efficacité d'un programme proposé basé sur l'approche interculturelle dans le 
développement de quelques compétences de la compréhension écrite chez les futurs enseignants de FLE. Thèse du Magistère .Université de Sohag.

- Blanchet, P. (2005). L'approche interculturelle en didactique du FLE . cours d'CED de didactique $\mathrm{du}$ français langue étrangère de $3^{\text {ème }}$ année de licences, service universitaire d'enseignement à distance. Université Rennes 2 Haute Bretagne. Tiré de WWW.aidenligne-françaisuniversité.auf.org/IMG/pdf_Blanc het_inter.pdf.

- Brouillet, P.(2002). La maréchaussée dans la généralité de Paris au xv111e siècle (17181797): étude institutionnelle et sociale. Thèse de doctorat en histoire moderne de la France, Paris-EPHE. Tiré dehttp://www. Thèses.fr/.

- Brno.2010. La gastronomie française. magisterská diplomová práce .Masarykova univerzita Fakulta. Katedra francouzského a literatury.

- Carlo, $\mathrm{C}$ et Causa, M. (2002).Civilisations progressives $\mathrm{du}$ français avec 300 activités. France : CLE International .

- Clanet, J.(1990). L'interculturel, introduction aux approches interculturelles en Education et Sciences Humaines. Toulouse: Presses Universitaires du Mirail.

- Covo.J et Fell.E- M. (2007). Cahiers de civilisation espagnole: l'enseignement de
civilisation,Paris :Rennes

Céeci.revue.org/114.

- Cuq, J-P. (2003). La civilisation. Dictionnaire de didactique du français langue étrangère et seconde. CLE internatinale. Tiré de WWW.cle inter.com(detail97820900339727-html).

- Desset, F. (2007). Mythes et légendes dans l'œuvre de Percy Bysshe Shelley: étude hypertextuelle de la poésie mythologique shelleyenne. Thèse de doctorat en Anglais, Tours. Tiré de http://www. Thèses.fr/.

- Dumas, J-P. (2006). L'administration du commerce et de l'industrie de 1870 à 1917: instituations publiques et tranformation de l'état. Thèse de doctorat en Histoire contemporaine de la France, France. Tiré de http://www. Thèses.fr/.

- Eia, M. (2006). Hypertextualité et recherche. Conception de l'information informatisée d'apprentissage de la formation Maltt au TECA. Tiré de http://tecfa.unige.ch/tecfa/teachin g/uv39/projets/0001/club_travail/ hypertexte.htm.

- Eid, T. (2013).Effet d'un programme basé sur l'utilisation des quelques stratégies de l'enseignement actif pour le développement de quelques notions culturelles et des compétences de l'expression orale et écrite en français chez les élèves du cycle secondaire. Thèse 
de Doctorat. Faculté de Pédagogie, Université de Minia.

- Fahim, S. (2005). Elaboration d'un modèle d'apprentissage fondé sur l'autonomie pour développer la compétence de la communication écrite en F.L.E comme deuxième langue étrangère chez les élèves au cycle secondaire, thèse de doctorat. Faculté de Pédagogie de Tanta. http://www.utc.fr/Nuntele.M.

- Gohard-Radenkovic, A.(2005). L'usage des concepts de «culture» et d' «interculturel» en didactique. Palaiseau: Les Éditions de l'École Polytechnique, p.23.

- Grand-clément, O. (2007). Civilisations en dialogues, Niveau débutant. France : CLE International.

- Meunier, O. (2007). Approches interculturelles en éduction, étude comparative

- internationale. Institut National de recherche Pédagogique. Service de veille scientifique et technologique. France : Lyon cedex.

- Mousa, A. (2012). Acquérir une compétence interculturelle en classe de langue, entre -objectifs visés, méthodes adoptées et Jordanien. Thèse de doctorat en sciences du langage et didactique des langues. Univérsité de lorraine, CREM (centre de recherche sur les méditations).
- Toureau, P. (2006). Civilisation .France. Tiré de www.toupie.org/Divers/licence.ht $\mathrm{m}$.

- Toureau, P. (2006). Interculturalité: Dictionnaire de politique, France. Tiré de WWW.Toupie.org/Divers/Licenc e.htm.

- Vanessa, D. (2012). Langue et interculturalité: Association Brasil Azur, Brésil, France.

- Yéo, 1. (2012). L'interculturel, facteur de paix et de cohésion sociale. Université Félix Houphouet-Boigny- CocodyAbidjan. Tiré deWWW.fabula/ actualités/ 1-interculturel.facterude-paix-et- de- cohésion sociale_53774.p.

\section{Site Internet:}

- La définition de la culture par l'UNESCO:

http://tpecultureadolescente.emonsite.com/pages/une-cultureadolescente-existe-t-elle/ladefinition-de-la-culture-par-1unesco.html. Gastronomie Françaisehttp://www.bu.edu/paris/ files/2011/01/GUIDE-DESCOURSES-.

- La définition de la gastronomie : https://fr.wikipedia.org/wiki/Gastr onomie.

- Eva.La cuisine française. Disponible sur https://ensemblefr.com/text2/LA_ CUISINE_FRANCAISE.pdf 